\title{
Pattern formation mechanisms in reaction-diffusion systems
}

\author{
VLADIMIR K. VANAG and IRVING R. EPSTEIN* \\ Department of Chemistry and Volen Center for Complex Systems, Brandeis University, Waltham, MA, USA
}

\begin{abstract}
In systems undergoing chemical reaction and diffusion, a remarkable variety of spatially structured patterns, stationary or moving, local or global, can arise, many of them reminiscent of forms and phenomena seen in living systems. Chemical systems offer the advantage that one can often control the parameters that determine the patterns formed and can thereby probe fundamental issues about pattern formation, with possible insights into biologically relevant phenomena. We present experimental examples and discuss several mechanisms by which such spatiotemporal structure may arise, classifying the mechanisms according to the type of instability that results in pattern formation. In some systems, the pattern that emerges depends not only on the chemical and physical parameters but also on the initial state of the system. Interactions between instabilities can result in particularly complex patterns.
\end{abstract}

KEY WORDS: pattern formation, reaction-diffusion, chemical kinetics, Turing pattern, wave instability

\section{Introduction}

Biology as a science probably began with efforts to systematize and classify the many varieties of plants and animals. The main criteria for such early taxonomic endeavors involved shapes or patterns. Clearly, biology in general, and developmental biology in particular, are closely linked to pattern formation. Recent progress in genomics points the way toward new approaches to systematization and taxonomy. Biological patterns are clearly dependent on the activity of genes. However, the path from genes to final patterns is long, tortuous and in most, perhaps all, cases, unknown, so that the conclusion that genes are the sole determinants of such processes may be premature. It seems reasonable to suggest that gene products generate patterns as components of self-organizing chemical networks, and the notion that preexisting patterns act to determine gene activation or expression is not outside the realm of possibility (Fields, 1996; Fields et al., 1997).

The term "patterns" in biology is perhaps too broad. One can consider external structures, for example, pigment spots on the skins of animals (Kondo and Asai, 1995), or, on the other hand, internal ones, like calcium waves inside living cells (Lechleiter et al., 1998). The mechanisms of pattern formation in biology are also extremely diverse. Structures like micelles or membranes can arise via equilibrium self-organization, while others, like the living cell itself, are stable only far from equilibrium and hence require an input of energy. Such structures can emerge from mechanical-chemical interactions or via growth starting from a newborn organism (Murray, 2003). One mechanism, or, more accurately, group of mechanisms, proposed by Alan Turing in a work entitled "The Chemical Basis of Morphogenesis" (Turing, 1952 ) is diffusive instability, which can be realized in homogeneous reaction-diffusion systems. It is this chemical-physical approach to biological pattern formation that we focus on here.

Of course, representing biological systems, which are typically quite heterogeneous, by a homogeneous reaction-diffusion medium is a rather crude approximation. Nevertheless, such an approach can give insights into real processes. Diffusive instabilities can provide the initial impetus for other mechanisms of pattern formation that dominate the later stages of patterning. In some cases, it appears that diffusive instabilities are the actual sources of pattern formation in biological systems.

As examples, we point first to several cases of propagating waves: $\mathrm{Ca}^{2+}$ waves within cells (Lechleiter et al., 1998) and between cells, (Harris-White et al., 1998), and NADH waves in living neutrophils, (Kindzelskii and Petty, 2002; Petty and Kindzelskii, 2001). A classic, but still extremely important example of wave propagation is the action potential in neurons

Abbreviations used in this paper: AOT, aerosol OT (sodium bis(2-ethylhexyl) sulfosuccinate); BZ, Belousov-Zhabotinsky; CDIMA, chlorine dioxide-iodinemalonic acid; NADH, nicotinamide adenine dinucleotide; SS, steady state; WNT, a protein signaling pathway.

\footnotetext{
*Address correspondence to: Irving R. Epstein. Department of Chemistry, Brandeis University, Waltham, MA 02454, USA. Fax: +1- 781-736-2516. e-mail: epstein@brandeis.edu
} 

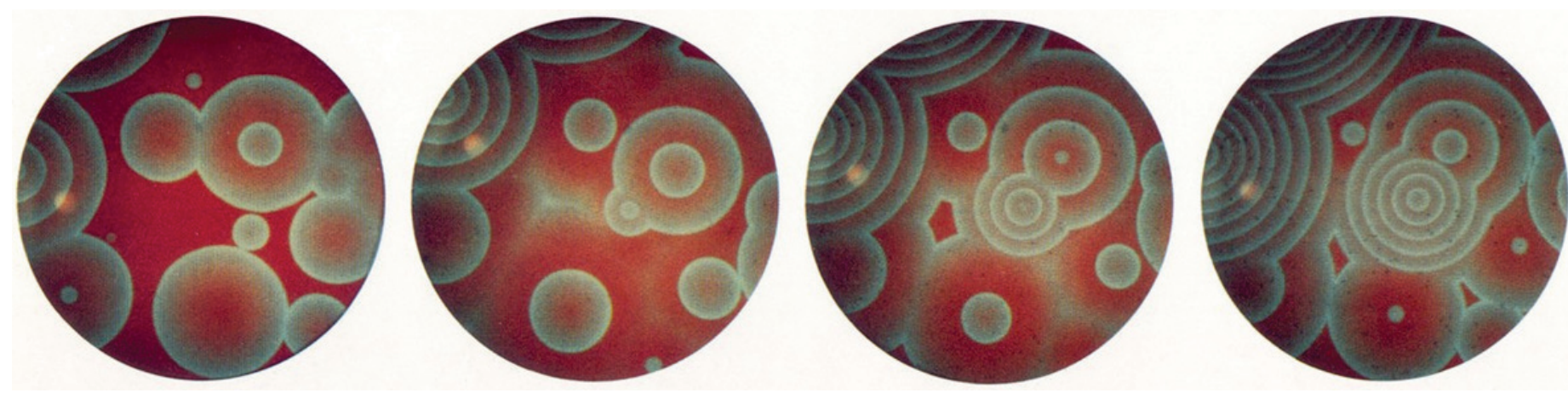

Fig. 1. Sequence of snapshots of target patterns in the aqueous Belousov-Zhabotinsky (BZ) reaction. Patterns emerge from an initially homogeneous red solution. Catalyst/indicator is ferroin. Red areas are more reduced; blue areas are more oxidized.

(Hodgkin and Huxley, 1952a; Hodgkin and Huxley, 1952b). The development of pigment spots on the skins of animals or fish has been suggested to occur via a Turing instability (Kondo, 2002; Kondo and Asai, 1995; Liu et al., 2006). Although the actual molecules and detailed mechanism involved in pigment pattern formation are not yet known, this "Turing mechanism" is certainly more plausible than the mechanism suggested by Rudyard Kipling for development of the spots on a leopard (Kipling, 1996). Very recently, a comprehensive Turing mechanism for hair follicle spacing, including the WNT signaling pathway, was suggested (Sick et al., 2006). The Turing mechanism, which involves socalled long range inhibition and short range activation (Gordon and Beloussov, 2006), can be applied even at the level of describing the behavior of a colony of ants (Theraulaz et al., 2002).

The main goal of this review is to present mechanisms of pattern formation in homogeneous reaction-diffusion chemical systems under controllable conditions, including concentrations, external signals, size of the system, temperature, and even diffusion coefficients. The advantage of chemical systems over biological ones in this regard is that we can manipulate almost all the parameters that affect patterning. In addition to presenting examples of reaction-diffusion patterns and an overview of mechanisms, we highlight examples in which the pattern obtained depends on the initial conditions in order to emphasize that physical-chemical principles alone may be insufficient to predict the spatiotemporal behavior of a complex living system. We take as our prototype system the Belousov-Zhabotinsky (BZ) oscillat-
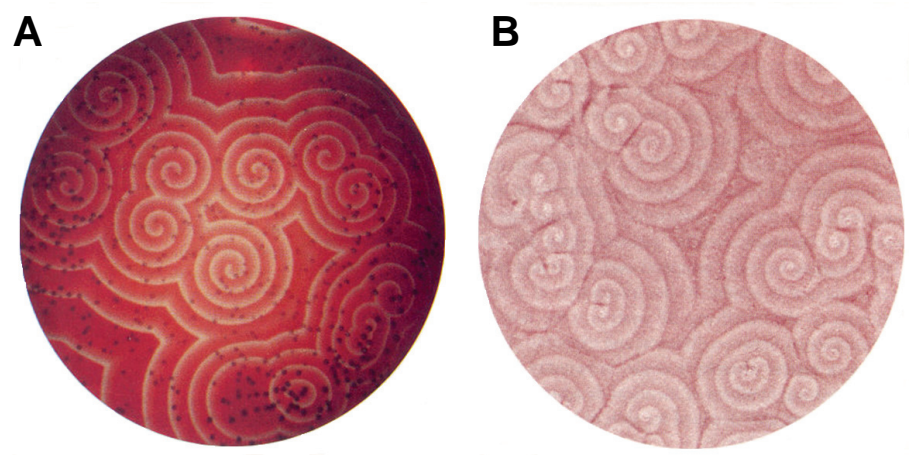

Fig. 2. Spiral patterns. (A) BZ reaction. (B) Aggregating Dictyostelium discoideum during aggregation of single cells in the process of spore formation induced by starvation. ing chemical reaction incorporated into nano-droplets of water in a reverse aerosol OT (AOT) microemulsion. Such a complex system can generate different patterns not only on varying the physical environment (droplet size or concentration of droplets) but even under the same conditions but starting from a slightly different initial state.

\section{Classification}

Taking as our starting point the initially uniform, unpatterned steady state, we can identify two types of instabilities that lead to pattern formation. For one class, an infinitesimal inhomogeneous perturbation can result in patterning. For the second, a finite perturbation, whose amplitude exceeds some threshold, is required to initiate pattern formation. The first category of instabilities includes supercritical wave instability and Turing instability, as well as Hopf instability (Cross and Hohenberg, 1993). These instabilities lead to global patterns that occupy the entire medium. The second kind consists of subcritical instabilities (wave, Turing, and Hopf), as well as excitability, the phenomenon whereby small perturbations of the initial steady state quickly decay but superthreshold disturbances first grow and cause a large excursion before the system returns to the initial (stable) state. Systems with two or more stable steady states can also belong to this second class. In some cases, instabilities of the second class can lead to localized patterns, which occupy only a small portion of the medium, but in other cases they yield global patterns like those produced by instabilities of the first type.

The classification of spatiotemporal instabilities is based on consideration of how a small, inhomogeneous perturbation applied to the homogeneous steady state evolves. The perturbation is assumed to be periodic in space (one can consider it as a Fourier component of a perturbation with a more general shape) and to grow or decay exponentially in time. We can then write it in the form $\varepsilon \exp (\lambda t+i k x)$, where $\varepsilon$ is the amplitude, $k$ is the wavenumber (inversely proportional to the wavelength), $x$ is the spatial coordinate, $i=(-1)^{1 / 2}$, and $t$ is time. The sign of $\lambda$ determines whether an infinitesimal perturbation with a particular $k$ grows, in which case the steady state is unstable, or shrinks, which would correspond to a stable steady state.

We can obtain $\lambda$ from the reaction-diffusion equations, the set of partial differential equations that describe the chemical kinetics and the diffusion in the system, by linear stability analysis (Nicolis and Prigogine, 1977). The process consists of writing each 
concentration variable $c_{j}$ as

$$
c_{j}(x, t)=c_{0}+\varepsilon_{j} \exp (\lambda t+i k x)
$$

where $c_{0}$ is the space- and time-independent steady state concentration of species $j$, and $\varepsilon$, is the (very small) amplitude of the perturbation in that species. We substitute eq. (1) for the concentrations in the reaction-diffusion equations and drop all terms of second order or higher in the $\varepsilon_{p}$, because the product of two infinitesimal quantities is negligibly small. We are left with a set of Nhomogeneous linear equations in Nunknowns, where $N$ is the number of species. These equations have a nontrivial (concentrations not all equal to zero) solution only for certain values of $\lambda$, the eigenvalues of the matrix of coefficients of the linear equations.

The dependence of $\lambda$ on $k$, called the dispersion relation, tells us much about how the system can behave. If $\lambda$ is negative for all $k$, then any small perturbation will decay, and the homogeneous steady state is stable. If $\lambda$ is real and has a positive maximum at some $k_{\text {max }}>0$, then perturbations with wavelengths at or near $2 \pi /$ $k_{\text {max }}$ tend to grow, and we have Turing instability, which can lead to patterns that are stationary in time and periodic in space (Castets et al., 1990; Turing, 1952; Vanag and Epstein, 2001b). If $\lambda$ is complex and $\operatorname{Re}(\lambda)$ is positive at some $k>0$ but negative at $k=0$, then we have wave instability, which can generate various wave patterns such as standing or traveling waves, packet or accelerating waves (Turing, 1952; Vanag and Epstein, 2001b;Vanag and Epstein, 2002). If $\operatorname{Re}(\lambda)$ is positive and $\operatorname{Im}(\lambda) \neq$ 0 at $k=0$, then we have Hopf instability, which leads to uniform, bulk oscillations (Field and Burger, 1985; Hopf, 1942).

It is possible for a system to possess more than one of these instabilities. Combinations of instabilities can produce striking patterns like the segmented spiral waves seen in experiments (Vanag and Epstein, 2003b) or the "pinwheels" found in computer simulations (Yang and Epstein, 2003b).

\section{Experimental systems}

The most thoroughly characterized and most versatile chemical system that exhibits pattern formation is the $B Z$ reaction, the metal-ion catalyzed oxidation of malonic acid or a similar organic species by bromate in a highly acidic, usually sulfuric acid, aqueous solution (Belousov, 1959; Zhabotinsky, 1964). At appropriate concentrations, the stirred reaction exhibits nearly periodic temporal oscillations in redox potential and in the concentration of many species, including bromide ion, that can persist for an hour or more even in a closed system like a beaker. Unstirred, e.g., in a petri dish, the system produces traveling concentration waves, typically concentric circles ("target patterns") or spirals that move out from a central "pacemaker" (Winfree, 1972; Zaikin and Zhabotinsky, 1970). These waves travel at constant velocity and, if two waves collide, they annihilate one another, often leaving intricate patterns. Fig. 1 shows an example of target patterns in an aqueous BZ system, while Fig. 2 compares spiral BZ patterns with those seen in an aggregating slime mold, Dictyostelium discoideum.

Although many systems exhibit bulk oscillations in a continuously fed stirred tank reactor (Sagues and Epstein, 2003), i.e., an open system, relatively few show sustained oscillation in a closed system or display pattern formation. In addition to the aqueous BZ reaction, three chemical systems have been found to exhibit a rich variety of patterns. These are the chlorite-iodide-malonic acid (CDIMA) reaction, (De Kepper et al., 1982; Lengyel et al., 1990), the BZ-AOT system (Vanag and Epstein, 2001a; Vanag and Epstein, 2001b; Vanag and Epstein, 2003b) and the ferrocyanideiodide-sulfite (FIS) reaction (Edblom et al., 1986; Lee et al., 1993; Lee et al., 1994).

The most prolific of these, in which nearly all known patterns have been found, is the BZ-AOT system, i.e., the BelousovZhabotinsky reaction in a water-in-oil microemulsion. The latter consists of nanometer diameter water droplets dispersed in the oil phase (octane, for instance). This system has several features analogous to living cells. All the chemical reactions, which involve the polar, hydrophilic reactants, occur inside the water droplets, but two hydrophobic intermediates, $\mathrm{Br}_{2}$ and $\mathrm{BrO}_{2}{ }^{\circ}$, can diffuse into and through the oil phase. They play the role of signaling molecules that transmit information from one droplet to another. The
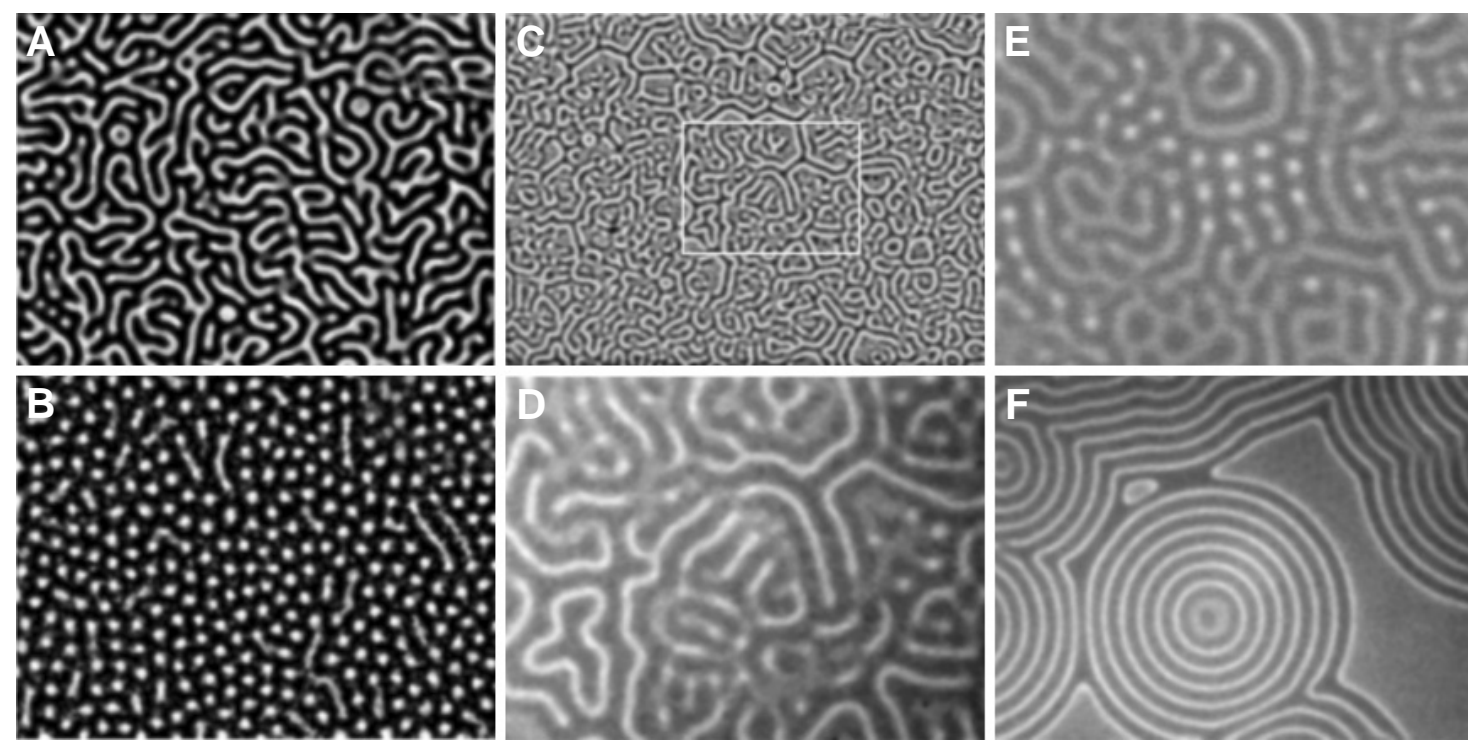

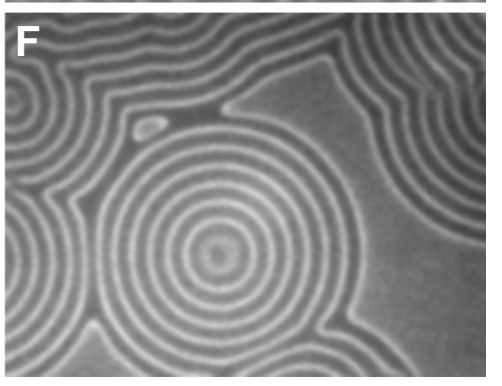

Fig. 3. Turing patterns in the BZ-AOT system. Initial concentrations (in M) in the aqueous phase and parameters $\omega$ and $\varphi_{d}$ of the AOT microemulsion: (A-F) $[\mathrm{MA}]=0.25 ;\left[\mathrm{H}_{2} \mathrm{SO}_{4}\right]=$ (A,B) $0.2,(C, D) 0.175$, (E) $0.14,(\mathrm{~F}) 0.142 ;\left[\mathrm{NaBrO}_{3}\right]=$ $(A, B) 0.18,(C, D) 0.15,(E)$ 0.14 , (F) 0.142 ; [ferroin] $=$ (A-F) $4.2 \times 10^{-3}$, $\left[R u(b p y)_{3}^{2+}\right]=(e) 0.25 \times$ $10^{-3}$ and 0 in all other cases, $R u(b p y)_{3}{ }^{2+}$ was added to make the BZ-AOT system photosensitive; $\omega=(A, B)$ 15, (C,D,F) 18.3, (E) 18; $\varphi_{d}$ $=(A, B) 0.355,(C-F) 0.48$;

frame size, $\mathrm{mm} \times \mathrm{mm},(A, B, F) 5 \times 3.75 ;(C) 7.6 \times 5.7 ;(D, E) 2.61 \times 1.96$. (A) 10 min after initiation; (B) 50 min after initiation; (D) is an enlargement of the white rectangle in frame (C). White corresponds to maximum value of catalyst $Z$ (ferriin) concentration, black to minimum. 

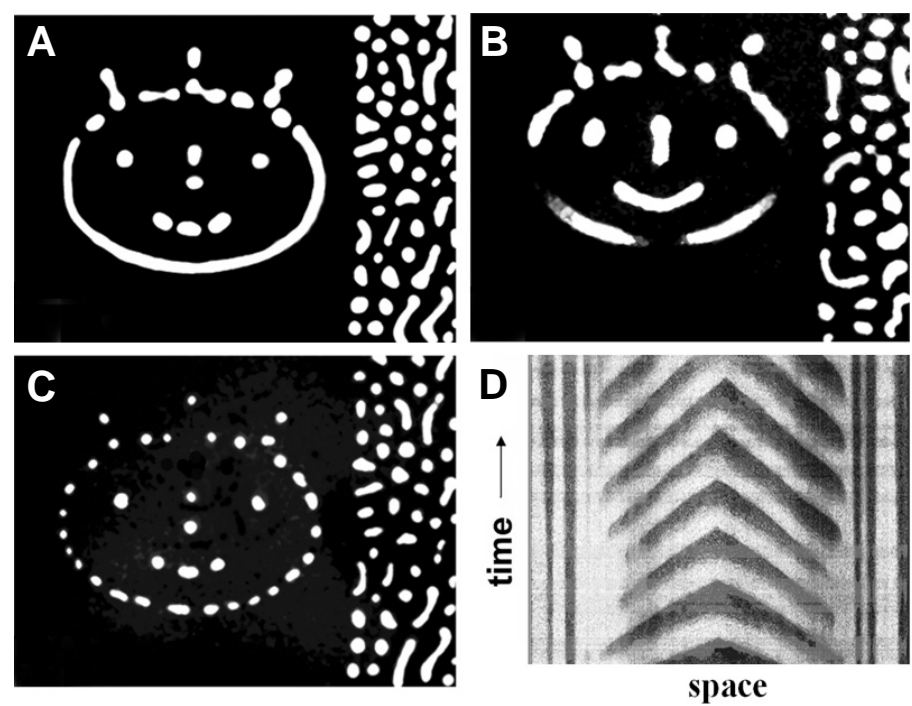

Fig. 4. Chemical memory. (A,B) stationary patterns. (C) Stationary spots and localized moving waves. Right section of the reactor is not illuminated, and stationary Turing patterns develop. After the image of the mask emerges, the mask is removed and the left section of the reactor is uniformly illuminated with intensity $\mathrm{I}=\mathrm{I}_{0} / 5, \mathrm{I}_{0}=28 \mathrm{~mW} / \mathrm{cm}^{2}$, while the right section of the reactor is not illuminated. (D) Space-time plot. Cross section is made along the lower oval of the mask image. Size $=10.8 \mathrm{~mm}$ $\times 18 \mathrm{~min}$. The thickness $\mathrm{h}$ of the image lines is $0.25 \mathrm{~mm} .\left[\mathrm{H}_{2} \mathrm{SO}_{4}\right]_{0}=0.3$ $\left.\mathrm{M}_{1}[\mathrm{NaBrO}]_{0}=0.25 \mathrm{M},[\mathrm{MA}]_{0}=0.1 \mathrm{M},[R u(b p y)]_{3}\right]_{0}=4 \mathrm{mM}, \omega=10, \varphi_{d}$ $=0.45$, Figures in left column are adapted from (Kaminaga et al., 2006) while figures in right column are unpublished results of Kaminaga, Vanag, and Epstein.

characteristic time of this "information transfer" over a distance / is determined by the diffusion coefficient $D$ of small molecules in octane $\left(\cong 2 \times 10^{-5} \mathrm{~cm}^{2} / \mathrm{s}\right)$ as $/ 2 / D$. Droplets can also collide, merge for a while, and then fission. This process also leads to mass or information exchange, but the rate of this process is significantly smaller, with characteristic time $/ 2 / D_{d}$, where $D_{d}$ is the diffusion coefficient of a droplet as a whole, which is dependent on the radius of the droplet through the StokesEinstein equation and is typically in the range $10^{-6}-10^{-7}$ $\mathrm{cm}^{2} / \mathrm{s}$ for this system.

To illustrate the variety of patterns in reaction-diffusion systems, we show here several examples, most of them found in the BZ-AOT system.

\section{Turing patterns}

Diffusive instabilities usually require a special relationship between diffusion coefficients. Ordinarily, for Turing instability the diffusion coefficient of the inhibitor, $D_{\text {inh }}$, should be significantly larger than that of the activator, $D_{\text {act }}$. This theoretical relation applies, however, only in the absence of cross-diffusion (Vanag and Epstein, 2009), i.e. the diffusion of each species must depend only on its own concentration gradient and not on gradients of other species, and it is clearly an oversimplification in systems with more than two variables, which include essentially all biological systems. In most real systems there are many variables, and it is often difficult or impossible to find a model that adequately explains the phenomenon of interest and possesses only two variables. Nevertheless, the insight from theory that a system that exhibits Turing-type behavior should have at least one fast-diffusing species that serves as an inhibitor, i.e., prevents or hinders autocatalysis, remains valid. In the BZAOT system, we can identify such a species in $\mathrm{Br}_{2}$. Bromine diffuses rapidly in the oil phase and inhibits autocatalysis through its facile conversion into $\mathrm{Br}^{-}$, which is the actual inhibitor in the $\mathrm{BZ}$ reaction.

Many different Turing patterns have been found in the BZ-AOT system; several are shown in Fig. 3. All the patterns in Fig. 3 are stationary, even those in Fig. 3F that resemble the concentric ring wave patterns in Fig. 1. In Fig. 3 we see spots (B), stripes (F), and labyrinthine (D) patterns. Mixtures of stripes and spots are also possible (E).

Turing patterns do not occur in the aqueous BZ system, because the condition of a fast-diffusing inhibitor cannot be realized, since all the (small molecule) species in this system have nearly equal diffusion coefficients. Introduction of the microemulsion in the BZ-AOT system makes it possible to control or regulate the rate of diffusion of $\mathrm{Br}_{2}$ relative to that of the aqueous species, because the size of the droplets, and hence their diffusion coefficient, depends in a simple fashion on the $\left[\mathrm{H}_{2} \mathrm{O}\right] /[\mathrm{AOT}]$ ratio, while the spacing between droplets depends upon [octane]/[H $\left.\mathrm{H}_{2} \mathrm{O}\right]$

If the metal catalyst used is the photosensitive ruthenium trisbipyridyl $\left[\mathrm{Ru}(\mathrm{bpy})_{3}{ }^{2+}\right]$, the light intensity can be employed as a further controllable parameter. In general, light of wavelength near $450 \mathrm{~nm}$ acts as a suppressor and can completely inhibit the $B Z$ reaction, as well as pattern formation, due to photogeneration of bromide (Krug et al., 1990). It is possible to tune the light $t=0$

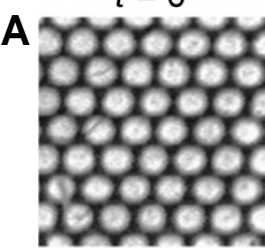

B

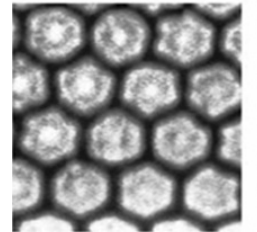

c

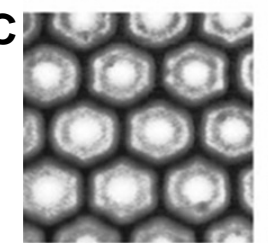

15
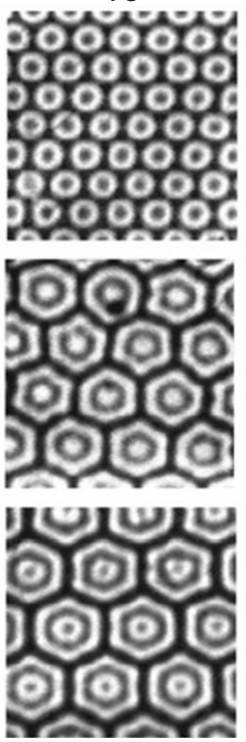

30
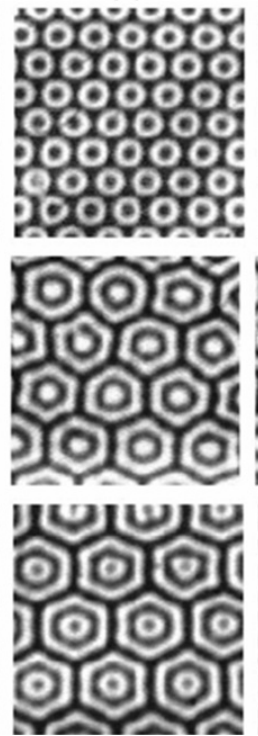

120

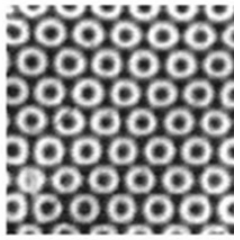

이은 ग1010 이인 시잉

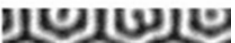

Fig. 5. Stationary Turing patterns in the CDIMA reaction developed after illumination through a mask. The ratio between the characteristic wavelengths of the mask and the native Turing patterns are equal to 2, 3 and 4, respectively for rows $(\mathbf{A}, \mathbf{B}, \mathbf{C})$. The numbers above the columns show the time (in $\mathrm{min}$ ) after cessation of illumination. Frame size $=5 \times 5 \mathrm{~mm}^{2}$. From Yang et al., (2006a) 

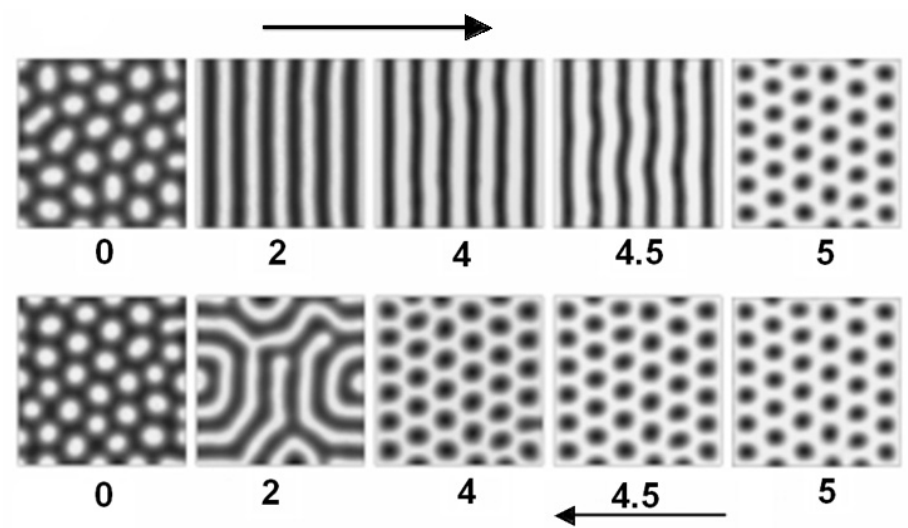

Fig. 6. Simulation of the photosensitive LE model (2), (3). Bistability between stripes and black spots at I $=4$ and 4.5. From Vanag and Epstein (2003c).

intensity in such a way that the BZ-AOT system is close to the onset of Turing instability. Under these conditions the BZ-AOT system displays hysteresis and bistability between a homogeneous steady state and stationary Turing patterns (Kaminaga et al., 2006).

In the dark, Turing patterns form spontaneously in this system. If the light intensity, /, is slowly increased, the patterns initially change only slightly, but at a critical intensity, Isc, they suddenly vanish, and the homogeneous steady state (SS) is seen. If we then slowly decrease /, the Turing patterns spontaneously reemerge at a lower intensity, $I_{c}$. For $I_{c}</<I_{\text {sc }}$, the system is bistable, a characteristic feature of subcritical Turing instability. In this bistable range of /, localized patterns generated by a local perturbation can be stable, and Turing patterns cannot emerge spontaneously elsewhere in the medium, since the SS is also stable. In Fig. 4 A,B we show how the system can "memorize" the image of a mask. This example demonstrates an important general phenomenon, the ability of a dissipative nonlinear system to display different patterns depending upon its initial conditions.

At slightly different conditions, this system generates localized waves in addition to stationary localized spots. A snapshot of the mask memorized by the system is shown in Fig. 4C. The upper part of the image consists of stationary spots or short line segments, while the bottom part of the oval face is represented now by localized waves that can be clearly seen in Fig. 4D, a space-time plot made along the oval of the face image. The central point in Fig. 4D, where the waves collide and annihilate, corresponds to the lowest point of the face in Fig. 4C. Here we see that a single system can exhibit three qualitatively different behaviors: silent area (stationary homogeneous steady state), localized stationary spots and localized traveling waves.

This multifaceted behavior is not unique to the BZ-AOT system. The CDIMA system, in which Turing patterns were first discovered (Castets et al., 1990), is also capable of producing different Turing patterns under the same chemical and physical conditions. Fig. 5 shows a result analogous to the above experiment, where a spatially extended CDIMA reaction is briefly illuminated through different masks (left column), the masks and the light source are removed, and Turing patterns then develop in the dark (Yang et al., 2006a). In the rightmost column of Fig. 5 we see quite different stable complex Turing patterns belonging to the class of superlattices, patterns that have at least two different characteristic wavelengths. It is important to note that such patterns can arise only under conditions for which a thin layer of the CDIMA reactants produces labyrinthine Turing patterns.

Bistability between different types of Turing patterns was also found in computer simulations with a photosensitive version of the Lengyel-Epstein model of the CDIMA reaction (Lengyel and Epstein, 1991; Vanag and Epstein, 2003c)

$$
\begin{aligned}
& \partial u / \partial t=a-u-4 u v\left(1+u^{2}\right)-m(x, t)+\Delta u \\
& \partial v \partial t=\sigma b\left[u-u v\left(1+u^{2}\right)+m(x, t)\right]+\sigma d \Delta v
\end{aligned}
$$

In Fig. 6 we observe two different Turing patterns, stripes and black spots, which can coexist at the same parameters starting from different initial conditions.

Another important feature of patterns in dissipative, e.g., reacting systems, which has received insufficient attention in the literature, is that the morphology of a pattern may not provide much information about the mechanism that produces it. To illustrate this point, we show in Fig. 7 three labyrinthine stationary patterns; $(A)$ and $(C)$ are from experiments, while $(B)$ was generated by computer simulation. Pattern $(B)$ is not completely stationary, since the growth of the "tentacles" has not yet finished, but the central part of the labyrinth is no longer changing with the growth at the periphery, and this labyrinthine pattern will eventually become stationary. The patterns in Figs. 7(A) and $(B)$ developed in bistable systems that do not have a Turing instability, $(A)$ in the FIS reaction (Lee et al., 1993) and (B) in the FitzHugh-Nagumo model (Vanag and Epstein, 2007), while pattern (C) is a Turing pattern in the BZ-AOT system (Vanag and Epstein, 2001b). Another example of a computationally obtained labyrinthine pattern can be found in Fig. 6 (bottom row, second column). Both theory and experiment confirm that labyrinthine patterns can be obtained either in a bistable system without Turing instability or in a monostable system with Turing instability.

Our analysis of patterns in reaction-diffusion systems suggests that the shape and dynamical behavior of almost all patterns can be explained by at least two different mechanisms. This situation resembles that found in living creatures, where two
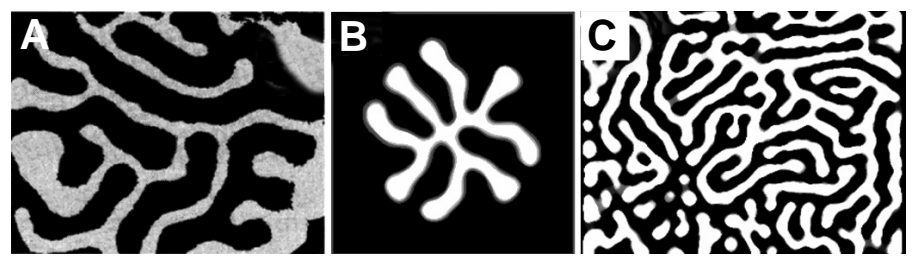

Fig. 7. Labyrinthine stationary patterns. (A) in the $\mathrm{Fe}(\mathrm{CN})_{6}^{4-}-1 \mathrm{O}_{3}^{-}$ $\mathrm{SO}_{3}{ }^{2-}$ system in a thin layer of polyacrylamide gel in a continuously fed unstirred reactor; light stripes correspond to low $\mathrm{pH}$, black stripes to high pH [from (Lee et al., 1993)], (B) in a bistable FitzHugh-Nagumo model (u - map): $\partial \mathrm{u} / \partial \tau=-\mathrm{u}(\mathrm{u}-\mathrm{a})(\mathrm{u}-1)-\mathrm{b}(\mathrm{v}-\mathrm{u})+\mathrm{D}_{u} \nabla^{2} \mathrm{u} ; \partial \mathrm{v} / \partial \tau=(-\mathrm{v}+\mathrm{u}) / \varepsilon+\mathrm{D}_{\mathrm{v}} \nabla$ ${ }^{2} \mathrm{v}$. White corresponds to $\mathrm{u}=1$, black to $\mathrm{u}=0$. Parameters $\mathrm{D}_{\mathrm{u}}=0.01, \mathrm{a}=$ $0.4, \mathrm{~b}=0.2,1 / \mathrm{D}_{\mathrm{v}}=\varepsilon=0.1$. Total size $=30 \times 30$. Initial circular perturbation with radius $\mathrm{R}_{0}=10$ shrinks and then slowly transforms to localized labyrinthine pattern [from (Vanag and Epstein, 2007)]. (C) in the BZ-AOT system. $\omega=15, \phi_{d}=0.35$. Gray levels quantify [ferroin], with white corresponding to minimum and black to maximum. Concentrations (M): $\left[\mathrm{H}_{2} \mathrm{SO}_{4}\right]_{0}=0.2,\left[\mathrm{NaBrO}_{3}\right]_{0}=0.17,[\mathrm{MA}]_{0}=0.3$. From Vanag and Epstein (2001b). 

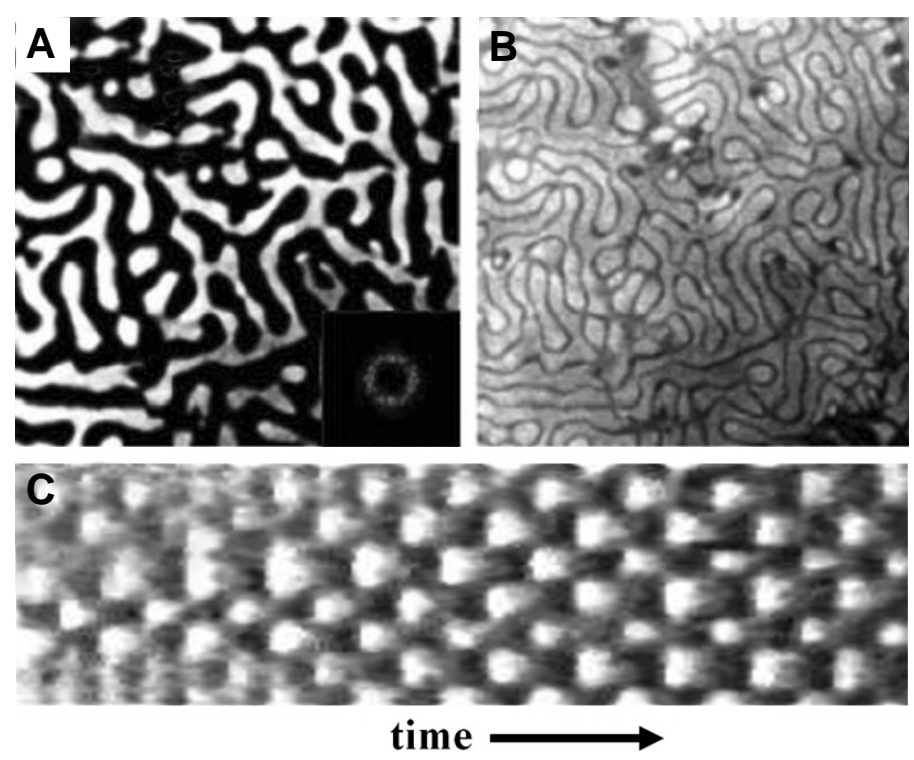

Fig. 8. Standing waves. (A) Snapshot, with spatial Fourier transform shown as inset. (B) Superposition of two snapshots taken half an oscillation period apart. (C) Space-time plot. From Kaminaga et al. (2005b).

organisms with similar appearance may have very different genomes, while two superficially different individuals may possess closely related genomes.

\section{Wave instability}

Next, we consider patterns that emerge due to wave instability, the second major diffusive instability. The first experimental evidence of wave instability in reaction-diffusion systems appeared only rather recently (Vanag and Epstein, 2001a; Vanag
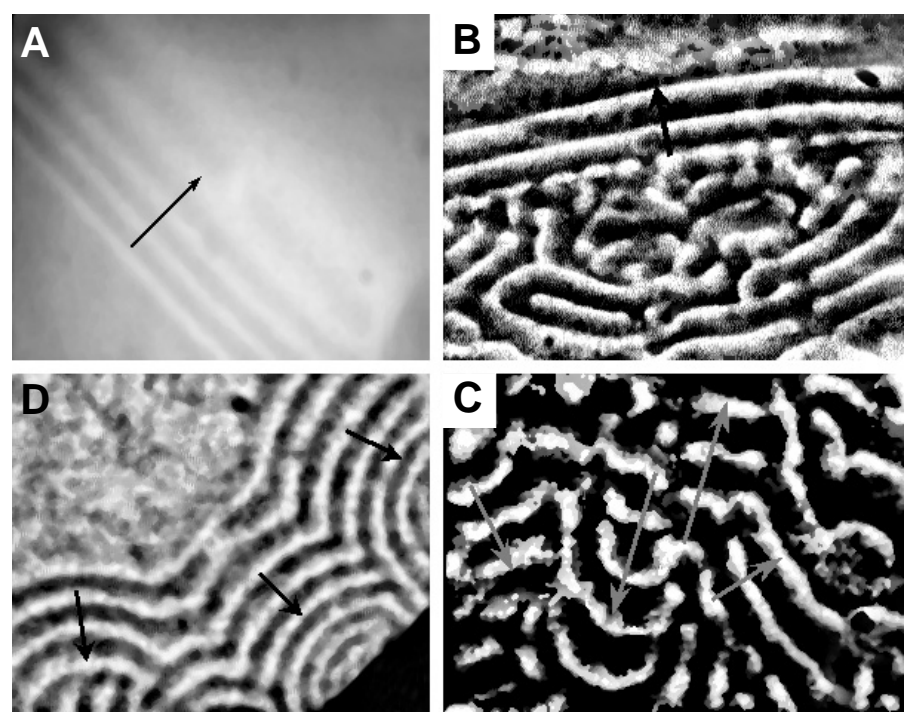

and Epstein, 2001b; Vanag and Epstein, 2002). Wave instability can manifest itself as standing waves (Fig. 8), as packet waves (Fig. 9), or as traveling waves with the special property that they collide without annihilation. This feature of traveling phase waves associated with a wave instability is not unique, however. Trigger waves, which arise via excitability, and which typically have a much larger amplitude, can also collide without annihilation, if the system is close to a subcritical Hopf bifurcation (Kosek and Marek, 1995). For trigger waves, non-annihilation is rare event, while for traveling phase waves supported by a wave instability, it is the rule. Although it is sometimes difficult to distinguish trigger and phase waves, the latter cannot occur in an excitable medium since they require periodic oscillations at each spatial point; phase waves arise from different phases of oscillation at different spatial points.

In the case of wave instability, each spatial point of the system oscillates with period $T_{w}$. Such a system typically gives rise to phase waves. Unlike phase waves in a system with a Hopf bifurcation, where the wavelength, which is determined by the initial concentration gradient, can take any value, here there is a characteristic wave length $L_{w}$ given by the dispersion relation $\lambda(k)$ as $L_{\mathrm{w}}=2 \pi / k_{\max }$, where $k_{\max }$ is the wavenumber at which $\operatorname{Re}(\lambda)$ attains its positive maximum. The velocity of the phase waves is $V_{p}=L_{w} / T_{w}$ where the period of oscillation $T_{w}$ is also determined from the dispersion curve as $T_{w}=2 \pi / \operatorname{lm}\left(\lambda_{w}\right)$, and $\lambda_{w}$ is the eigenvalue of the linear stability matrix at $k_{\max }$.

If we have a wave packet, like the ones shown in Fig. 9A, then the phase waves can propagate only within each packet. The packet itself can also propagate and spread with time. The velocity of the wave packet, the so-called group velocity, is given by $V_{\mathrm{g}}=\mathrm{dlm}(\lambda) / \mathrm{d} k$ at $k=k_{\max }$. This group velocity can be positive or negative. The latter case, in which individual waves and the packet as a whole move in opposite directions, gives rise to unusual phenomena, such as the antispirals found in experiments on the BZ-AOT system (Vanag and Epstein, 2001a). These waves propagate inwardly, toward the spiral center, while in normal spirals, which usually consist of trigger waves in an excitable medium, the waves propagate out from the center.

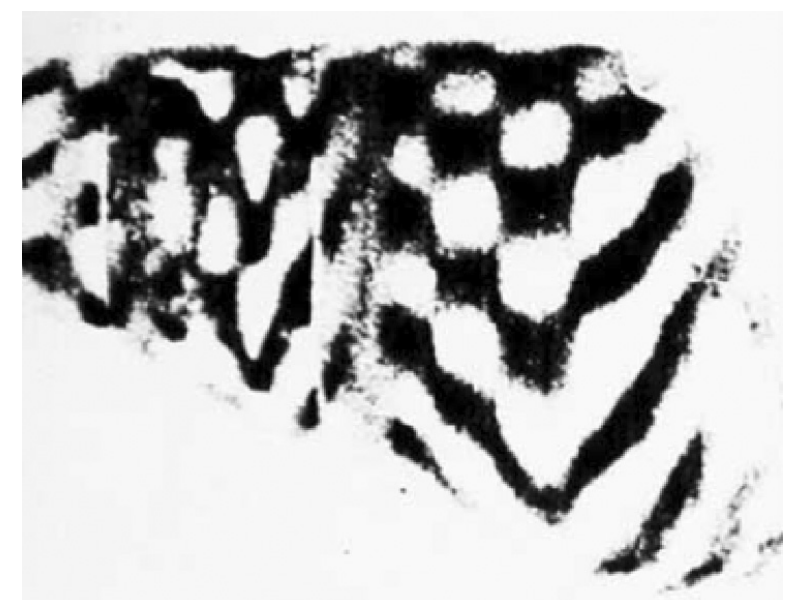

Fig. 9 (Left). Packet waves. (A) $[\mathrm{H} 2 \mathrm{O}] /[\mathrm{AOT}]=15$. (B,C) $[\mathrm{H} 2 \mathrm{O}] /[\mathrm{AOT}]=16.4$, (D) $[\mathrm{H} 2 \mathrm{O}] /[\mathrm{AO} T]=15.2 . \mathrm{Size}(\mathrm{mm} \times \mathrm{mm})(\mathrm{A}) 2.5 \times 2.2,(B, C) 1.88 \times$ 1.4, (D) $3.76 \times 2.81$. Turbulent wave pattern in (C) evolves from more regular pattern in (B). White corresponds to maximum catalyst (ferriin) concentration, black to minimum. Arrows mark direction of wave movement. From Vanag and Epstein (2002).

Fig. 10 (Right). Checkerboard-like pigment pattern on the shell of the small sea snail Bankivia fasciata. From Meinhardt (2004). 


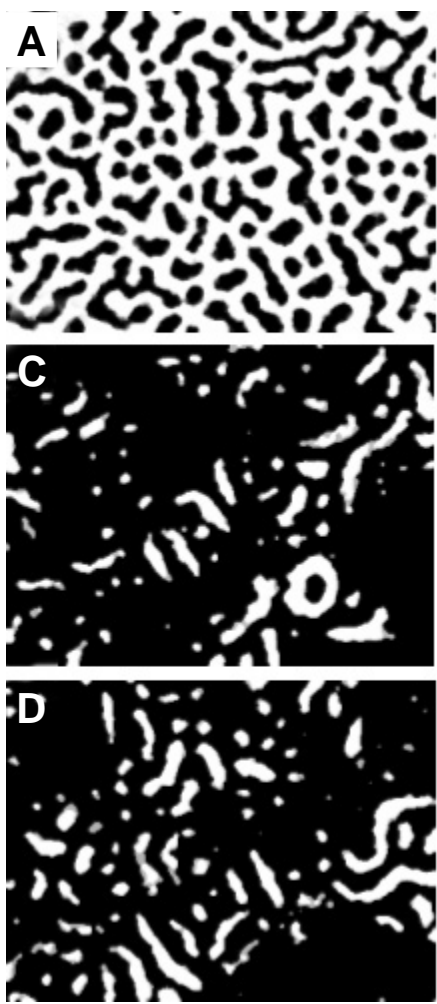

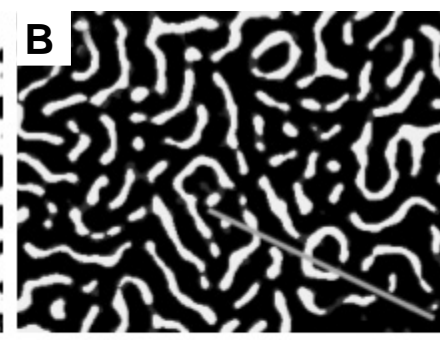

E

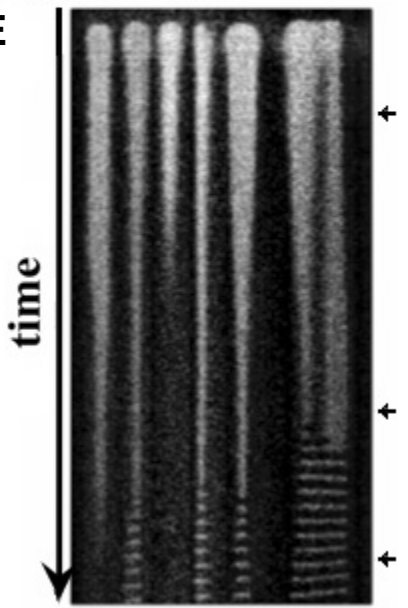

space
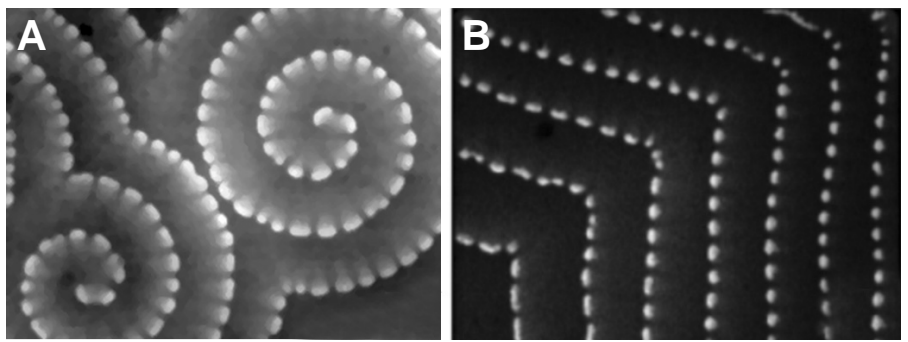

Fig. 12. Structured waves. Segmented spirals (A) from Vanag and Epstein (2003b) and dashed waves (B) in the BZ-AOT system.

found thus far only in the BZ-AOT system, probably arise from interaction between excitability and Turing instability (Vanag and Epstein, 2003a; Vanag and Epstein, 2003b). We can imagine at least two possible realizations of such an interaction. In one case, the system is bistable, and one steady state, SSI, is excitable while the other steady state, SSII, is Turing unstable. Here, excitability produces a trigger wave that switches the system into SSII, so the system can be in SSII only at those points that belong to a wave or pulse, i.e., locally. If such an interpretation applies to dash-waves, then dash-waves are a 1D moving Turing pattern. In the second case, the system is monostable with the single steady state possessing both excitability and a subcritical Turing or wave instability. Such a combination occurs in a modified FitzHughNagumo model (Bode etal., 2002; Schenk etal., 1997), which can produce not only dash-waves (Vanag and Epstein, unpublished), but jumping waves (Yang et al., 2006b) as well, in which a pulse periodically disappears from its original position and then reemerges at a fixed distance. Such jumping waves have recently been found experimentally.

The BZ-AOT system catalyzed by bathoferroin exhibits a remarkable example of bimodal behavior, in which two qualitatively different patterns, segmented waves and jumping waves, can coexist in the same system (Fig. 13A). This system is quite sensitive to small changes in parameters and, as key concentrations evolve over time, it exhibits a sequence of dynamical behaviors analogous to development in an organism. For example, an initial pattern of normal spiral waves progresses to segmented spirals or to the combination of segmented spirals and jumping waves seen in Fig. 13A and then to a novel pattern, the "jumping bubbles" shown in Fig. 13B. Ultimately, the system "dies," reaching the uniform steady state.

Interaction between Turing and wave instabilities can, at least theoretically, produce a rich array of hybrid patterns, yielding various combinations of spots, stripes and spirals as seen in Fig.

\section{Patterns involving interacting instabilities}

Two or more different instabilities, Turing, Hopf, wave, and excitability, can interact and produce even more complex patterns. Recently, oscillatory Turing patterns were found both in the BZ-AOT system (Kaminaga et al., 2005a) (see Fig. 11) and in the CDIMA reaction (Míguez etal., 2006). These patterns are the result of interaction between Turing and Hopf instabilities. One can imagine a situation in which it would be quite difficult, from experimental observations alone, to distinguish oscillatory Turing patterns from standing waves.

Segmented spirals and dash-waves (Fig. 12),
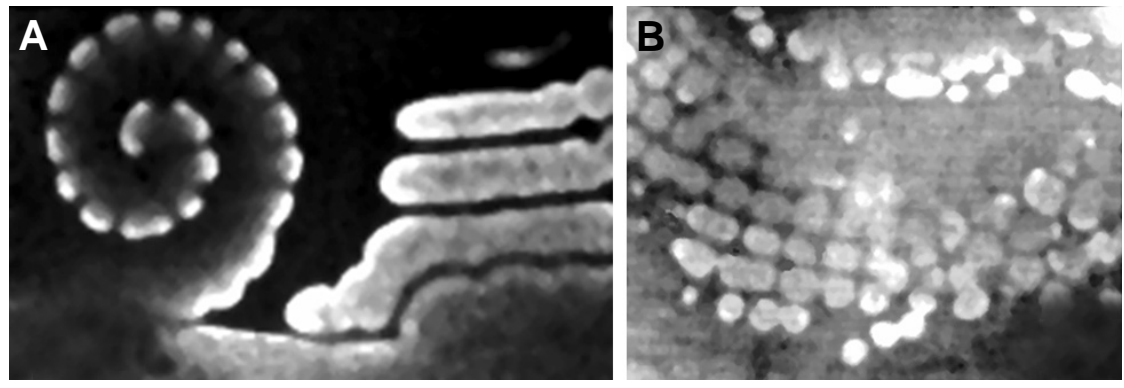

Fig. 13. The BZ-AOT system. (A) Segmented spiral and jumping waves. (B) Jumping bubbles. From Vanag (2004). 

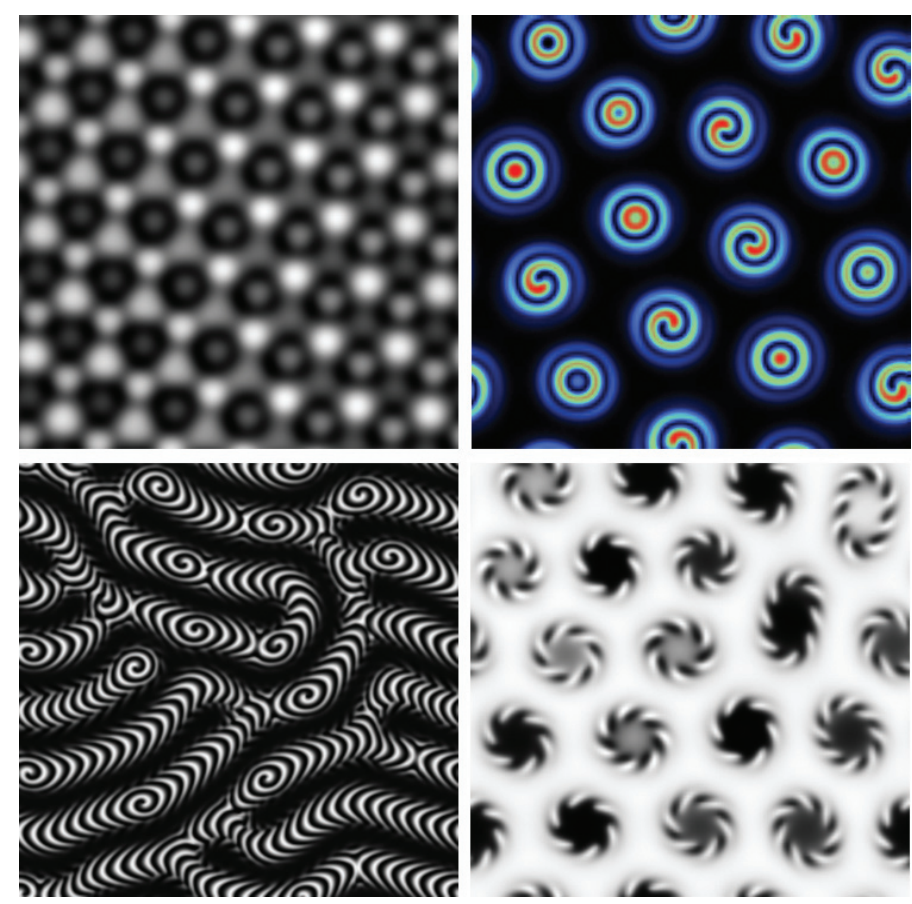

Fig. 14. Hybrid patterns resulting from interaction between Turing and wave instabilities in a model of two coupled layers. From Yang and Epstein (2003a).

14 (Yang and Epstein, 2003a). Interaction between a pair of Turing instabilities can occur in bistable and in multilayer systems and is thought to be responsible for "black eye" and "white eye" superlattice patterns (Bachir et al., 2001; Yang et al., 2002).

\section{Conclusion}

Reaction-diffusion systems can produce a diverse and beautiful array of spatial and spatiotemporal patterns, many of which resemble structures and phenomena seen in living systems. These patterns are observed and studied both in relatively simple experimental systems, which allow for considerable control over the system parameters, and in mathematical models. Interestingly, many of these systems display a form of multistability, in which the same set of parameters can yield two or more qualitatively different behaviors or patterns, and the one that is ultimately selected depends upon the initial conditions or the past history of the system.

It is important to note that, in all cases of which we are aware, any single pattern can be explained by at least two different mechanisms. Thus, while models may yield important insights into the origin of pattern formation, one should be wary of the "same pattern implies same mechanism" fallacy. Proposed mechanisms require extensive testing by all available experimental means. The only way to decide which of two plausible mechanisms is "better" is to apply both mechanisms over a range of conditions, not just to a single experiment, and see which one's predictions are robust.

The richness of behavior generated by relatively simple chemical systems like the BZ-AOT system is truly impressive. On the one hand, it offers some hope that we can begin to understand pattern-forming process in biology by looking at simpler, more easily controlled systems. On the other hand we see that an increase of the number of species involved in pattern formation mechanism leads to more complex patterns. For example, patterns generated by wave instability like standing waves (Fig. 8), require three variables and cannot arise in two-variable models. Experiments and modeling with two coupled layers, i.e., four or five variable systems, demonstrate even richer behavior due to interaction between different instabilities (Fig. 14). If a handful of inorganic chemicals can already generate such an immense variety, the range of possibilities available to the molecules of life seems almost unimaginable. Nevertheless, the results obtained in our "simple" systems and their striking similarity to living patterns suggest that chemistry may have much to teach us about the constraints that ultimately determine the range of biologically accessible patterns.

\section{Acknowledgments}

We thank ourmany able coworkers, includinglgalBerenstein, Alexander Cherkashin, Milos Dolnik, Akiko Kaminaga, Lingfa Yang and Anatol Zhabotinsky. This work was supported by the National Science Foundation under grants CHE-0615507and CHE-0526866.

\section{References}

BACHIR, M., MÉTENS, S., BORCKMANS, P., and DEWEL, G. (2001). Formation of rhombic and superlattice patterns in bistable systems. Europhys. Lett. 54: 612-618.

BELOUSOV, B.P. (1959). A periodic reaction and its mechanism. In Collection of Short Papers on Radiation Medicine, pp. 145-152. Moscow, Medgiz.

BODE, M., LIEHR, A.W., SCHENK, C.P., and PURWINS, H.-G. (2002). Interaction of dissipative solitons: particle-like behaviour of localized structures in a threecomponent reaction-diffusion system. IPhysica D 161: 45-66.

CASTETS, V., DULOS, E., BOISSONADE, J., and DE KEPPER, P. (1990). Experimental Evidence of a Sustained Standing Turing-Type Nonequilibrium Chemical Pattern. Phys. Rev. Lett. 64: 2953-2956.

CHERKASHIN, A.A., VANAG, V.K., and EPSTEIN, I.R. (2008). Discontinuously propagating waves in the bathoferroin-catalyzed Belousov-Zhabotinsky reaction incorporated into a microemulsion. J. Chem. Phys. 128: 204508.

CROSS, M.C. AND HOHENBERG, P.C. (1993). Pattern formation outside of equilibrium. Rev. Mod. Phys. 65: 851-1112.

DE KEPPER, P., EPSTEIN, I.R., KUSTIN, K., and ORBÁN, M. (1982). Batch Oscillations and Spatial Wave Patterns in Chlorite Oscillating-Systems. J. Phys. Chem. 86: 170-171.

EDBLOM, E.C., ORBÁN, M., and EPSTEIN, I.R. (1986). A New lodate Oscillator the Landolt Reaction with Ferrocyanide in a CSTR. J. Am. Chem. Soc. 108: 2826-2830.

FIELD, R.J. and BURGER, M., eds. (1985). Oscillations and Traveling Waves in Chemical Systems. New York, Wiley.

FIELDS, R.D. (1996). Signaling from neural impulses to genes. Neuroscientist 2 315-325.

FIELDS, R.D., ESHETE, F., STEVENS, B., and ITOH, K. (1997). Action potentialdependent regulation of gene expression: Temporal specificity in $\mathrm{Ca}^{2+} \mathrm{cAMP}$ responsive element binding proteins, and mitogen-activated protein kinase signaling. J. Neurosci. 17: 7252-7266.

GORDON, R. and BELOUSSOV, L. (2006). From observations to paradigms; the importance of theories and models. An interview with Hans Meinhardt. Int. J. Dev. Biol. 50: 103-111.

HARRIS-WHITE, M.E., ZANOTTI, S.A., FRAUTSCHY, S.A., and CHARLES, A.C (1998). Spiral intercellular calcium waves in hippocampal slice cultures. $J$ Neurophysiol. 79: 1045-1052.

HODGKIN, A.L. and HUXLEY, A.F. (1952a) A Quantitative Description of Membrane Current and Its Application to Conduction and Excitation in Nerve. $J$. Physiol.-London 117: 500-544. 
HODGKIN, A.L. and HUXLEY, A.F. (1952b) Propagation of Electrical Signals Along Giant Nerve Fibres. Proc. Roy. Soc. London Ser. B-Biol. Sci. 140: 177-183.

HOPF, E. (1942). Ber. Sachs. Akad. Wiss. MP94: 3.

KAMINAGA, A., VANAG, V.K., and EPSTEIN, I.R. (2005a). «Black spots» in a surfactant-rich BZ-AOT microemulsion system. J. Chem. Phys. 122: 174706.

KAMINAGA, A., VANAG, V.K., and EPSTEIN, I.R. (2005b). Wavelength Halving in a Transition between Standing Waves and Traveling Waves. Phys. Rev. Lett. 95: 058302.

KAMINAGA, A., VANAG, V.K., and EPSTEIN, I.R. (2006). A reaction-diffusion memory device. Angew. Chem. Int. Ed. 45: 3087-3089.

KINDZELSKII, A.L. and PETTY, H.R. (2002). Apparent role of traveling metabolic waves in oxidant realease by living neutrophils. Proc. Nat. Acad. Sci. USA99: 9207-9212.

KIPLING, R. (1996). Just So Stories. New York, William Morrow and Company.

KONDO, S. (2002). The reaction-diffusion system: a mechanism for autonomous pattern formation in the animal skin. Genes to Cells 7: 535-541.

KONDO, S. and ASAI, R. (1995). A Reaction-Diffusion Wave on the Skin of the Marine Angelfish Pomacanthus. Nature 376: 765-768.

KOSEK, J. and MAREK, M. (1995). Collision-Stable Waves in Excitable ReactionDiffusion Systems. Phys. Rev. Lett. 74: 2134-2137.

KRUG, H.J., POHLMANN, L., and KUHNERT, L. (1990). Analysis of the Modified Complete Oregonator Accounting for Oxygen Sensitivity and Photosensitivity of Belousov-Zhabotinsky Systems. J. Phys. Chem. 94: 4862-4866.

LECHLEITER, J.D., JOHN, L.M., and CAMACHO, P. (1998). Ca ${ }^{2+}$ wave dispersion and spiral wave entrainment in Xenopus laevis oocytes overexpressing $\mathrm{Ca}^{2+}$ ATPases. Biophys. Chem. 72: 123-129.

LEE, K.J., MCCORMICK, W.D., OUYANG, Q., and SWINNEY, H.L. (1993). Pattern-Formation by Interacting Chemical Fronts. Science 261: 192-194.

LEE, K.J., MCCORMICK, W.D., PEARSON, J.E., and SWINNEY, H.L. (1994). Experimental Observation of Self-Replicating Spots in a Reaction-Diffusion Systems. Nature 369: 215-218.

LENGYEL, I. and EPSTEIN, I.R. (1991). Modeling of Turing Structures in the Chlorite-lodide-Malonic Acid-Starch Reaction System. Science 251: 650-652.

LENGYEL, I., RÁBAI, G., and EPSTEIN, I.R. (1990). Batch Oscillation in the Reaction of Chlorine Dioxide with lodine and Malonic Acid. J. Am. Chem. Soc. 112: 4606-4607.

LIU, R.T., LIAW, S.S., and MAINI, P.K. (2006). Two-stage Turing model for generating pigment patterns on the leopard and the jaguar. Phys. Rev. E74: 011914.

MEINHARDT, H. (2004). Out-of-phase oscillations and traveling waves with unusual properties: the use of three-component systems in biology. Physica $D$ 199: 264-277.

MíGUEZ, D.G., ALONSO, S., MUÑUZURI, A.P., and SAGUÉS, F. (2006). Experimental evidence of localized oscillations in the photosensitive chlorine dioxideiodine-malonic acid reaction. Phys. Rev. Lett. 97: 178301

MURRAY, J.D. (2003). Mathematical Biology.II: Spatial Models and Biomedical Applications. New York, Springer.

NICOLIS, G. and PRIGOGINE, I. (1977). Self-Organization in Nonequilibrium Systems. New York, Wiley-Interscience.

PETTY, H.R. and KINDZELSKII, A.L. (2001). Dissipative metabolic patterns respond during neutrophil transmembrane signaling. Proc. Nat. Acad. Sci. USA 98: 3145-3149.
SAGUÉS, F. and EPSTEIN, I.R. (2003). Nonlinear chemical dynamics. Dalton Trans. 7: 1201-1217.

SCHENK, C.P., OR-GUIL, M., BODE, M., and PURWINS, H.-G. (1997). Interacting Pulses in Three-Component Reaction-diffusion Systems on Two-Dimensional Domains. Phys. Rev. Lett. 78: 3781-3784.

SICK, S., REINKER, S., TIMMER, J., and SCHLAKE, T. (2006). WNT and DKK determine hair follicle spacing through a reaction-diffusion mechanism. Science 314: 1447-1450.

THERAULAZ, G., BONABEAU, E., NICOLIS, S.C., SOLE, R.V., FOURCASSIE, V., BLANCO, S., FOURNIER, R., JOLY, J.L., FERNANDEZ, P., GRIMAL, A., DALLE, P., and DENEUBOURG, J.L. (2002). Spatial patterns in ant colonies. Proc. Nat. Acad. Sci. USA 99: 9645-9649.

TURING, A.M. (1952). The chemical basis of morphogenesis. Philos. Trans. $R$. Soc. London. Ser. B237: 37-72.

VANAG, V.K. (2004). Waves and patterns in reaction-diffusion systems. BelousovZhabotinsky reaction in water-in-oil microemulsions. Physics-Uspekhi47: 923941

VANAG, V.K. and EPSTEIN, I.R. (2001a). Inwardly Rotating Spiral Waves in a Reaction-Diffusion System. Science 294: 835-837.

VANAG, V.K. and EPSTEIN, I.R. (2001b). Pattern Formation in a Tunable Medium The Belousov-Zhabotinsky Reaction in an Aerosol OT Microemulsion. Phys. Rev. Lett. 87: 228301.

VANAG, V.K. and EPSTEIN, I.R (2002). Packet Waves in a Reaction-Diffusion System. Phys. Rev. Lett. 88: 088303.

VANAG, V.K. and EPSTEIN, I.R (2003a). Dash-waves in a reaction-diffusion system. Phys. Rev. Lett. 90: 098301.

VANAG, V.K. and EPSTEIN, I.R (2003b). Segmented Spiral Waves in a ReactionDiffusion System. Proc. Natl. Acad. Sci. USA 100: 14635-14638.

VANAG, V.K. and EPSTEIN, I.R (2003c). Translational and non-translationa motion of perturbed Turing patterns. Phys. Rev. E67: 066219.

VANAG, V.K. and EPSTEIN, I.R (2007). Localized patterns in reaction-diffusion systems. Chaos 17: 037110.

VANAG, V.K. and EPSTEIN, I.R. (2009). Cross-diffusion and pattern formation in reaction-diffusion systems. Phys. Chem. Chem. Phys. 11: 897-912.

WINFREE, A.T. (1972). Spiral Waves of Chemical Activity. Science 175: 634-636

YANG, L., DOLNIK, M., ZHABOTINSKY, A.M., and EPSTEIN, I.R. (2002). Spatial resonances and superposition patterns in a reaction-diffusion model with interacting turing modes. Phys. Rev. Lett. 88: 208303.

YANG, L. and EPSTEIN, I.R. (2003a). Oscillatory Turing Patterns in ReactionDiffusion Systems with Two Coupled Layers. Phys. Rev. Lett. 90: 178303.

YANG, L.F., DOLNIK, M., ZHABOTINSKY, A.M., and EPSTEIN, I.R. (2006a) Turing patterns beyond hexagons and stripes. Chaos 16: 037114.

YANG, L.F. and EPSTEIN, I.R. (2003b). Oscillatory Turing patterns in reactiondiffusion systems with two coupled layers. Phys. Rev. Lett. 90: 178303.

YANG, L.F., ZHABOTINSKY, A.M., and EPSTEIN, I.R. (2006b). Jumping solitary waves in an autonomous reaction-diffusion system with subcritical wave instability. Phys. Chem. Chem. Phys. 8: 4647-4651.

ZAIKIN, A.N. and ZHABOTINSKY, A.M. (1970). Concentration Wave Propagation in a Two-Dimensional, Liquid Phase Self-Oscillating System. Nature 225: 535

ZHABOTINSKY, A.M. (1964). Periodic liquid phase reactions. Proc. Acad. Sci. USSR 157: 392-395. 


\section{Further Related Reading, published previously in the Int. J. Dev. Biol.}

See our Special Issue Pattern Formation edited by Chen-Ming Chuong and Michael Richardson at: http://www.ijdb.ehu.es/web/contents.php?vol=53\&issue=5-6

See our Special Issue Developmental Morphodynamics edited by Lev Beloussov and Richard Gordon at: http://www.ijdb.ehu.es/web/contents.php?vol=50\&issue=2-3

Analyses of regenerative wave patterns in adult hair follicle populations reveal macroenvironmental regulation of stem cell activity

Maksim V. Plikus, Randall B. Widelitz, Rob Maxson and Cheng-Ming Chuong

Int. J. Dev. Biol. 53: doi: 10.1387/ijdb.072564mp (in press)

Before programs: The physical origination of multicellular forms

Stuart A. Newman, Gabor Forgacs and Gerd B. Müller

Int. J. Dev. Biol. (2006) 50: 289-299

The dynamic geometry of mass cell movements in animal morphogenesis

Vladimir G. Cherdantsev

Int. J. Dev. Biol. (2006) 50: 169-182

From observations to paradigms; the importance of theories and models. An interview with Hans Meinhardt

Richard Gordon and Lev Beloussov

Int. J. Dev. Biol. (2006) 50: 103-111

A moving wave patterns the cone photoreceptor mosaic array in the zebrafish retina Pamela A. Raymond and Linda K. Barthel

Int. J. Dev. Biol. (2004) 48: 935-945

Integument pattern formation involves genetic and epigenetic controls: feather arrays simulated by digital hormone models.

Ting-Xin Jiang, Randall B Widelitz, Wei-Min Shen, Peter Will, Da-Yu Wu, Chih-Min Lin, HanSung Jung and Cheng-Ming Chuong

Int. J. Dev. Biol. (2004) 48: 117-135

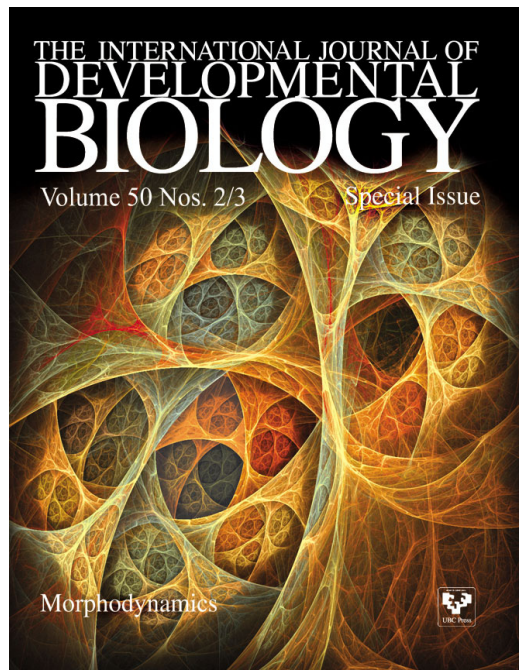

5 yr ISI Impact Factor $(2008)=3.271$

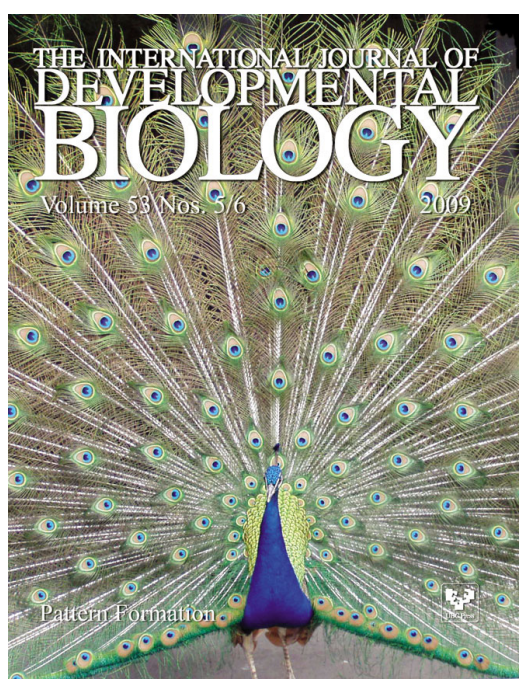

